\title{
SÍNTESE DO 2-(1H-INDOL-3-IL)-2-OXO-N-FENIL ACETAMIDA EM MICRORREATOR: VERIFICAÇÃO DA INFLUÊNCIA DO TEMPO DE RESIDÊNCIA
}

\author{
VINICIOS TORREZAN ALVES, JAILDA SANTOS VASCONCELOS, RAISA \\ CALIL, TAINÃ GARCIA, EVELIN FORNARI PEREIRA, MAURI SÉRGIO ALVES \\ PALMA
}

\author{
Universidade de São Paulo/Faculdade de Ciências Farmacêuticas \\ msapalma@usp.br
}

\begin{abstract}
Resumo
Neste trabalho foi estudada a síntese do 2-(1H-indol-3-il)-2-oxo-N-fenil acetamida, um composto derivado do Indol, e que tem potencial de ter atividade farmacológica, em um microrreator capilar. Foi verificada a influência do tempo de residência no reator na composição do produto de reação, tendo sido determinados, de modo preliminar as massas molares dos produtos em GC-MS e HPLC-MS. Testou-se a reação do Indol com Cloreto de Oxalila e Anilina em concentração de 0,2 M dissolvidos em THF em temperatura ambiente. Nesta etapa preliminar do estudo foram testadas 2 tempos de residência (30 e $60 \mathrm{~s}$ ).
\end{abstract}

\section{Introdução}

O microrreator é um dispositivo que possibilita reação química, tais como os reatores convencionais, mas com dimensões menores, por exemplo, canais com 10 a $150 \mu \mathrm{m}$. Os microrreatores permitem explorar a operação em novas condições de processo ("New Process Windows") como, por ex., temperaturas extremas e elevadas pressões, para aumentar o rendimento e seletividade das reações (Wiles e Watts, 2010; McQuade e Seeberger, 2013). Fazem parte de um campo de estudo que vem recebendo muita atenção da comunidade de engenharia química que é Engenharia de Micro Processos (Dudukovic, 2010).

Os microrreatores apresentam várias vantagens em relação ao reator batch como, pequeno tempo de mistura devido às pequenas distâncias para difusão (Watts e Wiles, 2012), alta conversão e seletividade (Yoshida et al., 2005), altos coeficientes de transferência de calor de até $6 \times 10^{4} \mathrm{~W} / \mathrm{m}^{2} . \mathrm{K}$ devido à alta relação superfície/volume, enquanto que para os reatores batch são da ordem de $100 \mathrm{~W} / \mathrm{m}^{2}$.K. Permite a realização de forma segura de reações altamente exotérmicas como nitrações, fluorações e diazotações (Baraldi et al, 2014), reações ocorrem em regime permanente com alta reprodutibilidade; pode-se estudar com facilidade os efeitos das variáveis de processo como a temperatura, tempo de residência e relação estequiométrica; pode-se determinar parâmetros cinéticos (Valera et al, 2010; Patel e Davis, 2010; Zaborenko et al., 2011); pode-se usar solventes voláteis à altas temperaturas, mantendo-os líquidos com elevadas pressões, o que facilita a sua remoção no final da reação, pode-se usar solventes em condição supercrítica; pode-se ativar reações fotoquímicas em microrreatores de vidro ou quartzo (Wiles e Watts, 2011); podem ser utilizados na síntese de pequenas quantidades de produtos, pesquisa e desenvolvimento e produção industrial; scale-up é mais seguro e pode ser feito através da paralelização ou aumento de diâmetro (Wiles e Watts, 2010; Zaborenko et al., 2011; Shirazi e Rolando, 2012), muitos ensaios podem ser realizados com pequenas quantidades de reagentes, da ordem de mg/ensaio, e em pouco tempo, da ordem de minutos/ensaio.

Os compostos com anéis heterocíclicos estão intimamente ligados aos processos bioquímicos básicos da vida. Se fosse escolhida uma etapa em uma síntese bioquímica aleatória teria uma boa chance de que um dos reagentes ou produtos seria um composto heterocíclico. Três dos vinte aminoácidos encontrados em enzimas contêm anéis heterocíclicos. 
O sucesso de um agente medicinal depende do equilíbrio entre os efeitos farmacológicos desejados e os danos que pode fazer a um paciente, e no entanto este não pode ser previsto com certeza.

O Indol (Figura 1A) é um derivado do Triptofano (Figura 1B), um aminoácido essencial e, como tal, é um componente da maior parte das proteínas.<smiles></smiles>

(A)<smiles>NC(Cc1c[nH]c2ccccc12)C(=O)O</smiles>

(B)

Figura 1 - Molécula de Indol (A) e de Triptofano (B).

O Indol é um alcaloide e é um composto orgânico aromático heterocíclico. Sua estrutura bicíclica é composta por um Pirrol, que caracteriza um anel de 5 átomos sendo um deles nitrogênio, com uma dupla ligação e acoplado a um anel benzênico, como visto na Figura 1.

O Indol e seus derivados podem ser sintetizados por uma variedade de métodos. As principais rotas industriais utilizam a anilina que é reagida, em fase vapor entre 200 e $500^{\circ} \mathrm{C}$, com o etilenoglicol na presença de um catalisador e com rendimento de até $60 \%$.

Neste estudo foi realizada a síntese do 2-(1H-indol-3-il)-2-oxo-N-fenil acetamida, obtido através de um composto intermediário derivado do Indol (2-(3H-indol-3-il)-2-oxoacetil cloro) e cuja reação está representada na Figura 2. Tal estudo faz parte do projeto de pesquisa intitulado "Microrreatores na Síntese de Fármacos" financiado pela FAPESP e ora no início de suas atividades práticas. Aquele projeto tem como objetivo identificar as melhores condições de temperatura, relação estequiométrica dos reagentes e tempo de residência no microrreator para a obtenção de moléculas orgânicas com potencial farmacológico.

(A)<smiles>O=C(Cl)C(=O)C1C=Nc2ccccc21</smiles>

(B)<smiles>Nc1ccccc1</smiles>

(C)

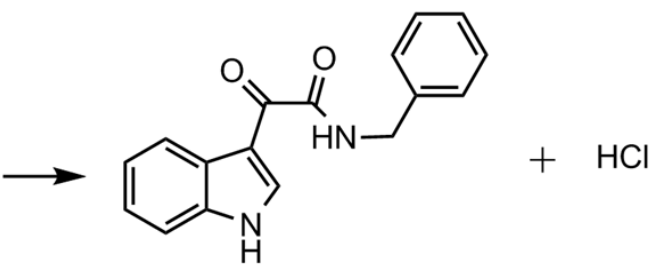

Figura 2 - Representação da reação: 2-(3H-indol-3-il)-2-oxoacetil cloro (A) + anilina (B) $\rightarrow$ 2-(1H-indol-3-il)2-oxo-N-fenil acetamida (C) + ácido clorídrico (D)

\section{Objetivos}

Este trabalho tem como objetivo estudar a síntese do produto (C) mostrado na Figura 2 em microrreator capilar fabricado pela Asia Inc. e verificar, neste estudo preliminar, a influência do tempo de residência na composição da mistura final da reação através de análises em GC-MS e HPLC-MS.

\section{Materiais e Métodos}

Na síntese do 2-(1H-indol-3-il)-2-oxo-N-fenil acetamida serão utilizados o composto intermediário 2(3H-indol-3-il)-2-oxoacetil cloro sintetizado através da reação do Indol com cloreto de oxalila, o qual será então reagido com anilina. Também serão utilizados: acetato de etila e $\mathrm{NH}_{4} \mathrm{Cl}$. Água destilada foi utilizada em todos os ensaios e amostragens. Os produtos químicos foram adquiridos da Sigma Aldrich e eram de grau P.A. O microrreator de vidro foi fornecido pela Asia e tinha volume de $250 \mu \mathrm{L}$. 
Na síntese do composto (C) serão determinadas, ao final do projeto financiado pela FAPESP, as melhores condições operacionais em termos de temperatura, tempo de residência no microrreator e relação estequiométrica dos reagentes. Na literatura encontram-se tempos de residência e temperaturas estudadas para outras reações de síntese de fármacos em microrreatores com grande variação, entre $5 \mathrm{~s}$ a 16 min e de $-25^{\circ} \mathrm{C}$ a $220^{\circ} \mathrm{C}$, respectivamente (Watts e Wiles, 2012).

O procedimento para obtenção do composto (C) consistiu em bombear ao reator 2 correntes contendo os dois reagentes dissolvidos em THF. A primeira corrente, de volume total $5 \mathrm{~mL}$, continha $0,2 \mathrm{M}$ de anilina, enquanto a segunda corrente, também de volume total $5 \mathrm{~mL}$, continha $0,2 \mathrm{M}$ do intermediário (A), previamente preparado através da reação de indol com cloreto de oxalila. A temperatura foi ambiente $\left(25^{\circ} \mathrm{C}\right)$ e as vazões testadas foram de 125 e $250 \mu \mathrm{L} / \mathrm{min}$ para cada corrente, correspondendo a tempos de residência de 60 e $30 \mathrm{~s}$, respectivamente. As amostras, de cerca de $1 \mathrm{~mL}$, eram coletadas em $\mathrm{NH}_{4} \mathrm{Cl}$ aquoso na saída do reator após 1 min do início do bombeamento das correntes. Adicionava-se às amostras cerca de $2 \mathrm{~mL}$ de acetato de etila para remover o produto e esta solução era então analisada em GC-MS e HPLC-MS.

\section{Resultados}

Os resultados das análises dos produtos de reação em GC-MS e HPLC-MS estão mostrados nas Figuras 3 e 4 .

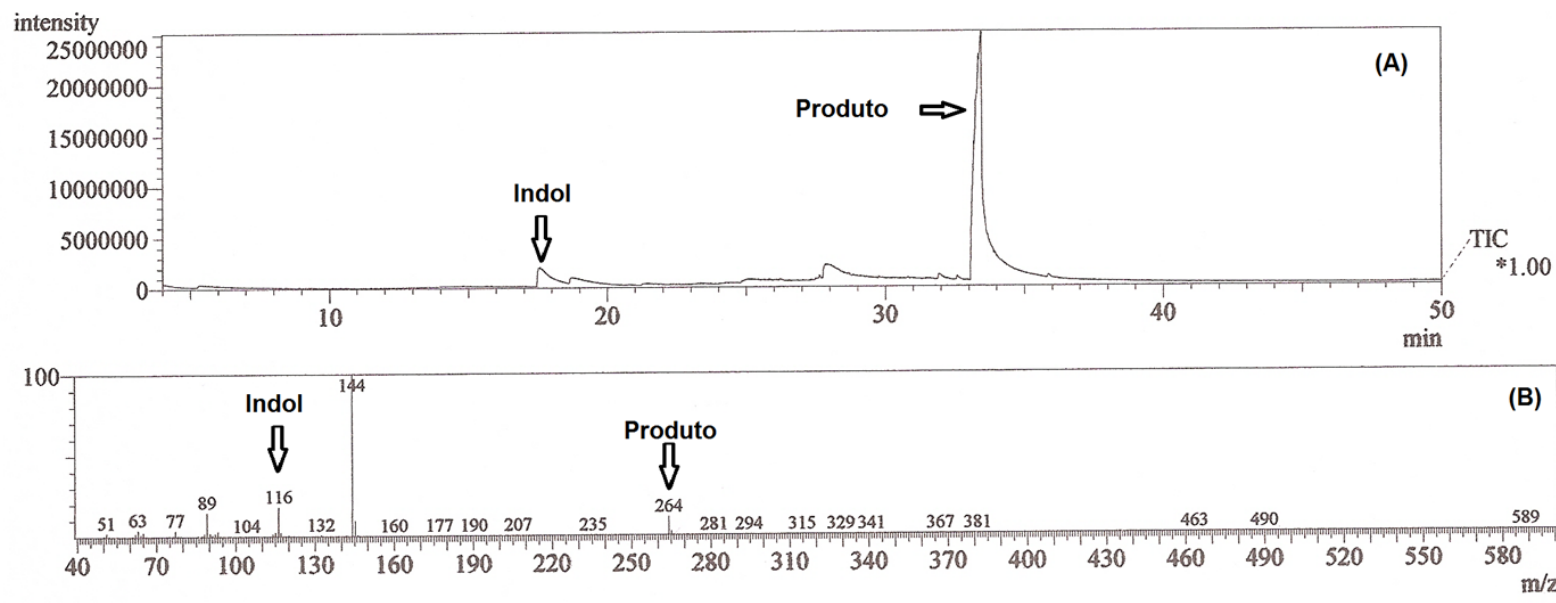

Figura 3 - Cromatograma da amostra analisada em GC-MS (Tempo de residência $=60 \mathrm{~s}$ ).

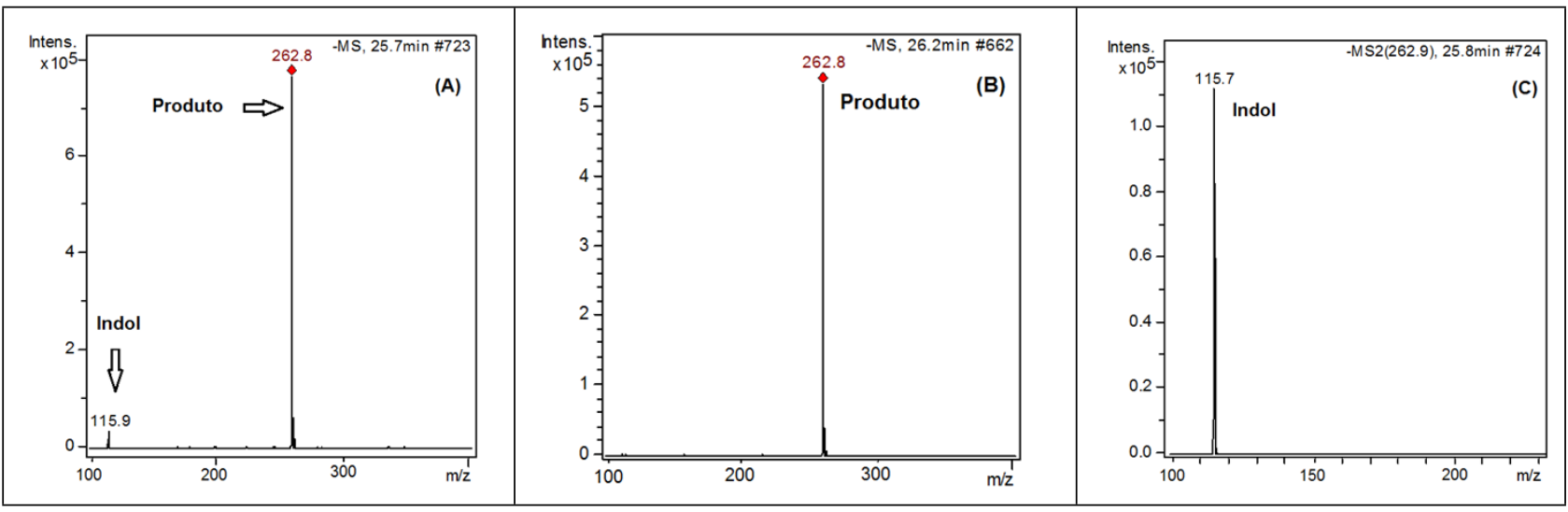

Figura 4 - Cromatograma das amostras analisadas em HPLC-MS. (A) Tempo de residência $=30 \mathrm{~s}$; (B) e (C) Tempo de residência $=60 \mathrm{~s}$.

A Figura 3A mostra a presença preponderante do indol e do produto, com tempos de retenção de 17,5 min para o indol e de 33 min par ao produto. Os resultados de espectrometria de massa mostrados na Figura 3B 
indicam a presença de substâncias com massas molares $89,116,144$ e 264 , das quais correspondem ao indol $(\mathrm{MM}=117)$ e do produto formado $(\mathrm{MM}=264)$.

A Figura 4A mostra que para tempo de residência no reator $60 \mathrm{~s}$ há indol e produto com tempos de retenção na coluna cromatográfica de $22,5 \mathrm{~min}$. As Figuras 4B e 4C mostramque para tempo de residência no reator de $30 \mathrm{~s}$ também se observa a presença de indol e de produto. No entanto, se observa que a intensidade do sinal do produto é $27 \%$ menor no ensaio com tempo de residência $30 \mathrm{~s}$, enquanto que a intensidade do sinal do indol é 3 vezes menor no ensaio com tempo de residência $60 \mathrm{~s}$. Isto indica que o rendimento e conversão da reação aumentaram com o tempo de residência de 30 para $60 \mathrm{~s}$.

\section{Conclusões}

Este estudo preliminar mostrou que o Microrreator capilar permite realizar reações com tempos de residência extremamente pequenos. A síntese testada neste trabalho foi realizada em batelada por Stefani et al. (2013) em tempos de reação de $2 \mathrm{hs.} \mathrm{Com} \mathrm{a} \mathrm{continuidade} \mathrm{deste} \mathrm{trabalho} \mathrm{será} \mathrm{feito} \mathrm{um} \mathrm{planejamento}$ fatorial para se determinar as melhores condições operacionais em termos de tempo de residência, temperatura e relação estequiométrica dos reagentes, que resultem na maior conversão, rendimento e seletividade do produto desejado.

\section{Agradecimentos}

Os autores agradecem o apoio do Prof. Helio Alexandre Stefani do Departamento de Farmácia da Faculdade de Ciências Farmacêuticas da USP pelo apoio na realização dos ensaios e das análises em GCMS.

\section{Referências Bibliográficas}

Baraldi, P. T.; Noel, T.; Wang, Q.; Hessel, V. The accelerated preparation of 1,4-dihydropyridines using microflow reactors. Tetrahedron Letters, v. 55, p. 2090-2092, 2014.

Dudukovic, M.P. Reaction engineering: Status and future challenges. Chemical Engineering Science, v. 65, p. 3-11, 2010.

McQuade, D. T.; Seeberger, P. H. Applying flow chemistry: methods, materials, and multistep synthesis. The Journal of Organic Chemistry, v. 78, p. 6384-6389, 2013.

Patel, M. K.; Davis, B. G. Flow chemistry kinetic studies reveal reaction conditions for ready access to unsymmetrical trehalose analogues. Organic \& Biomolecular Chemistry, v. 8, p. 4232-4235, 2010.

Stefani, H. A.; Vasconcelos, S. N. S.; Souza, F. B.; Manarin, F.; Zuckerman-Schpector, J. One-pot threecomponent synthesis of indole-3-glyoxyl derivatives and indole-3-glyoxyl triazoles. Tetrahedron Letters, v. 54, p. 5821-5825, 2013.

Valera, F. E,; Quarant, M.; Moran, A.; Blacker, J.; Armstrong, A.; Cabral, J. T.; Blackmond, D. G. The flow's the thing...or is it? Assessing the merits of homogeneous reactions in flask and flow. Angewandte Chemie Int. Ed., v. 49, p. 2478-2485, 2010.

Watts, P.; Wiles, C. Micro reactors, flow reactors and continuous flow synthesis. Journal of Chemical Research, n. 4, p. 181-193, 2012.

Wiles, C.; Watts, P. Continuous process technology: a tool for sustainable production. Green Chemistry, v. 16, p. 55-62, 2014.

Wiles, C.; Watts, P. Recent advances in micro reaction technology. Chemical Communications, v. 47, p. 6512-6535, 2011.

Wiles, C.; Watts, P. Continuous flow reactors: a perspective. Green Chemistry, v. 14, p. 38-54, 2012.

Wiles, C.; Watts, P. The scale-up of organic synthesis using micro reactors. Chemistry Today, v. $28, \mathrm{n}$. 3, 2010.

Yoshida, J.; Nagaki, A.; Iwasaki, T.; Suga, S. Enhancement of chemical selectivity by microreactors. Chemical Engineering \& Technology, v. 28 n. 3, p. 259, 2005.

Zaborenko, N.; Bedore, M. W.; Jamison, T. F.; Jensen, K. F. Kinetic and scale-up investigations of epoxide aminolysis in microreactors at high temperature and pressures. Organic Process Research \& Development, v. 15, p. 131-139, 2011. 